\title{
ATIVIDADE ANTIMICROBIANA DE MICRORGANISMOS ISOLADOS DE GRÃOS DE KEFIR
}

\section{ANTIMICROBIAL ACTIVITY OF MICROORGANISMS ISOLATED FROM KEFIR GRAINS}

\author{
Priscila Alves Dias ${ }^{1}$ \\ Daiani Teixeira Silva ${ }^{1}$ \\ Cláudio Dias Timm ${ }^{*}$ \\ ${ }^{1}$ Universidade Federal de Pelotas, Pelotas, RS, Brasil. \\ *Autor para correspondência - timm@ufpel.tche.br
}

\section{Resumo}

Kefir é o produto da fermentação do leite pelos grãos de kefir. Esses grãos contêm uma mistura simbiótica de bactérias e leveduras imersas em uma matriz composta de polissacarídeos e proteínas. Muitos benefícios à saúde humana têm sido atribuídos ao kefir, incluindo atividade antimicrobiana contra bactérias Gram positivas e Gram negativas. A atividade antimicrobiana de 60 microrganismos isolados de grãos de kefir, frente à Escherichia coli O157:H7, Salmonella enterica subsp. enterica sorotipos Typhimurium e Enteritidis, Staphylococcus aureus e Listeria monocytogenes, foi estudada através do teste do antagonismo. A ação antimicrobiana dos sobrenadantes das bactérias ácidolácticas que apresentaram atividade no teste do antagonismo foi testada. $\mathrm{O}$ experimento foi repetido usando sobrenadantes com pH neutralizado. Salmonella Typhimurium e Enteritidis sobreviveram por 24 horas no kefir em fermentação. E. coli $\mathrm{O} 157: \mathrm{H} 7$, S. aureus e L. monocytogenes foram recuperados até 72 horas após o início da fermentação. Todos os isolados apresentaram atividade antimicrobiana contra pelo menos um dos patógenos usados no teste do antagonismo. Sobrenadantes de 25 isolados apresentaram atividade inibitória e três mantiveram essa atividade com $\mathrm{pH}$ neutralizado. As bactérias patogênicas estudadas sobreviveram por tempo superior àquele normalmente utilizado para a fermentação do kefir artesanal, o que caracteriza perigo em potencial para o consumidor quando a matéria-prima não apresentar segurança sanitária. Lactobacillus isolados de grãos de kefir apresentam atividade antimicrobiana contra cepas de E. coli O157:H7, Salmonella sorotipos Typhimurium e Enteritidis, S. aureus e L. monocytogenes além daquela exercida pela diminuição do $\mathrm{pH}$.

Palavras-chave: ação antibacteriana; Lactobacillus; leite fermentado; patógenos.

\begin{abstract}
Kefir is the product of milk fermentation by kefir grains. These grains contain a mixture of symbiotic bacteria and yeasts embedded in a matrix composed of polysaccharides and proteins. Many human health benefits have been attributed to kefir, including antimicrobial activity against Gram positive and Gram negative bacteria. We studied the antimicrobial activity of sixty bacterial strains isolated from kefir grains compared to Escherichia coli O157:H7, Salmonella enterica subsp. enterica serotypes Typhimurium and Enteritidis, Staphylococcus aureus, and Listeria monocytogenes through antagonism test. We tested the antimicrobial activity of lactic acid bacteria supernatants that were active in the antagonism test. The experiment was repeated using supernatants with neutralized $\mathrm{pH}$.
\end{abstract}


Salmonella Typhimurium and Enteritidis survived for 24 hours in kefir fermentation. E. coli O157:H7, S. aureus and L. monocytogenes were recovered up to 72 hours after the start of fermentation. All isolates showed antimicrobial activity against at least one of the pathogens used in the test of antagonism. Supernatants of 25 isolates showed inhibitory activity, and three maintained this activity with neutralized $\mathrm{pH}$. Pathogenic bacteria studied survived for longer than the time normally used for fermentation of artisanal kefir, characterizing a potential danger to the consumer when the raw material does not provide health security. Lactobacillus isolated from kefir grains exhibit antimicrobial activity against strains of E. coli O157:H7, Salmonella serotypes Typhimurium and Enteritidis $S$. aureus, and L. monocytogenes in addition to that exerted by the lower $\mathrm{pH}$ values. Keywords: antibacterial action; fermented milk; Lactobacillus; pathogens.

Recebido em: 4 de abril de 2016

Aceito em: 08 de fevereiro de 2018

\section{Introdução}

Desde que os antibióticos passaram a ser utilizados em larga escala, as bactérias têm desenvolvido crescente capacidade de resistência. Devido a isso, esforços têm sido despendidos no sentido de desenvolver e estudar novos compostos que possam representar alternativas à terapia antibiótica convencional $^{(1)}$.

A identificação de cepas bacterianas com atividade antimicrobiana gera inúmeras possibilidades para linhas de pesquisa no sentido da aplicação prática dessas bactérias e das substâncias bioativas por elas produzidas. Seu uso não está restrito à terapêutica ou à medicina preventiva humana e veterinária. A agroindústria tem pela frente um grande desafio provocado pela restrição ao uso de antibióticos e quimioterápicos na produção de animais para o consumo humano. A tendência é que ocorra um avanço no uso de prebióticos e probióticos em substituição aos aditivos e promotores de crescimento. A indústria de alimentos também tem interesse na utilização de microrganismos com essas características como conservantes naturais, pois são capazes de inibir o desenvolvimento de bactérias deteriorantes e patogênicas, contribuindo para a melhoria das condições higiênico-sanitárias dos produtos.

Kefir é o produto da fermentação do leite pelos grãos de kefir. Esses grãos contêm uma mistura simbiótica de bactérias ácido-lácticas, ácido-acéticas e leveduras imersas em uma matriz composta de polissacarídeos e proteínas ${ }^{(2)}$. A composição microbiana dos grãos de kefir pode ser bastante variável, dependendo de diversos fatores, como a origem dos grãos e o modo de cultivo, sendo os gêneros mais frequentemente isolados Lactobacillus, Lactococcus, Leuconostoc, Enterococcus e leveduras como Saccharomyces e Kluyveromyces ${ }^{(3,4)}$.

Muitos benefícios à saúde humana têm sido atribuídos ao kefir, incluindo atividade antimicrobiana contra bactérias Gram positivas e Gram negativas ${ }^{(5-7)}$, o que tem levantado a possibilidade de se utilizarem alguns dos microrganismos que o compõem como probióticos ${ }^{(8,9)}$. Ácidos orgânicos, bacteriocinas e um exopolissacarídio (kefiran) produzidos pelas bactérias ácido-lácticas dos grãos de kefir são substâncias que têm sido responsabilizadas por suas propriedades antimicrobianas ${ }^{(10,11)}$.

O presente estudo teve como objetivo isolar dos grãos de kefir microrganismos com atividade antimicrobiana e verificar a importância da alteração do pH na ação inibitória. 


\section{Material e Métodos}

Grãos de kefir utilizados artesanalmente foram obtidos de três diferentes fontes e denominados para fins desse experimento, LIPOA CDT, LIPOA ABB e LIPOA MH. Para recuperação e restabelecimento dos grãos, eles foram adicionados a leite UHT desnatado, na proporção de 1:10, e incubados em banho-maria a $20^{\circ} \mathrm{C}$. Diariamente, o kefir foi coado utilizando-se coador estéril e os grãos novamente misturados ao leite, retornando à incubação. O processo foi repetido durante uma semana. Após esse período, os grãos foram separados por coagem e macerados com uso de almofariz esterilizado. Uma alíquota de $10 \mathrm{~g}$ foi homogeneizada com $90 \mathrm{~mL}$ de solução salina estéril $(0,9 \%$ de $\mathrm{NaCl}$ ) para semeadura por esgotamento em placas contendo ágar DeMan Rugosa Sharpe (MRS, Acumedia, Lansing, Michigan), as quais foram incubadas a $37{ }^{\circ} \mathrm{C}$ por 48 horas em aerobiose e anaerobiose para obtenção de colônias isoladas. Dez colônias aeróbicas e dez anaeróbicas, preferencialmente com morfologia distinta para cada tipo de kefir, foram transferidas para caldo MRS (Acumedia, Lansing, Michigan), incubadas a $37^{\circ} \mathrm{C}$ até turvamento do meio, misturadas com igual volume de glicerol a $80 \%$ em salina fosfatada tamponada (PBS 0,01 M, pH 7,4) e estocadas a $-70^{\circ} \mathrm{C}$. Quando necessário, os isolados foram recuperados em caldo MRS a $37^{\circ} \mathrm{C}$ por 48 horas.

Os isolados foram identificados de acordo com suas características morfológicas, coloração de Gram, catalase e fermentação de carboidratos, proposta por Barrow e Feltham ${ }^{(12)}$. Alguns isolados que não se enquadraram na classificação proposta por esses autores foram identificados através do kit GP Test Vitek $2^{\circledR}$ (BioMérieux) para bactérias Gram positivas.

Cepas de Salmonella enterica subsp. enterica sorotipos Typhimurium (ATCC 13311) e Enteritidis (ATCC 13076), Escherichia coli O157:H7 (ATCC 43895), Staphylococcus aureus (ATCC 14458) e Listeria monocytogenes (ATCC 7644) foram usadas como bactérias indicadoras. Quando necessário, as bactérias foram recuperadas em caldo de infusão de cérebro e coração (BHI, Oxoid, Basingstoke, Hampshire, England) a $37^{\circ} \mathrm{C}$ por 24 horas.

A atividade antimicrobiana foi estudada através do teste do antagonismo, segundo Jacobsen et al. ${ }^{(13)}$. Dois microlitros de cultura de cada isolado foram dispostos isoladamente na superfície de ágar MRS e incubados por 24 horas a $37^{\circ} \mathrm{C}$ para desenvolvimento de colônias, sob aerobiose ou anaerobiose, conforme as características de crescimento dos isolados. Duzentos microlitros de cultura overnight de cada bactéria indicadora foram misturados a $12 \mathrm{~mL}$ de BHI contendo $0,75 \%$ de ágar e espalhados sobre a superfície das placas com ágar MRS. As placas foram incubadas a $37^{\circ} \mathrm{C}$. Após 24 horas de incubação, foram lidas as zonas de inibição. A presença de zona clara com $1 \mathrm{~mm}$ ou mais, indicando área sem crescimento da bactéria indicadora ao redor das colônias dos isolados, foi considerada como resultado positivo. Os testes foram realizados em triplicata.

A separação do sobrenadante foi realizada de acordo com Lin et al. ${ }^{(14)}$. Culturas dos isolados em caldo MRS com 48 horas de incubação foram centrifugadas a $7000 \mathrm{rpm}$ por 5 minutos e o sobrenadante foi filtrado através de filtro estéril com poros de 0,22 $\mu \mathrm{m}$ (Millipore, Bedford, Massachusetts).

O método utilizado foi a microdiluição em caldo em placas de microtitulação, segundo Krüger et al. ${ }^{(15)}$. Cada cavidade das placas de microtitulação foi preenchida com $100 \mu \mathrm{L}$ de BHI, $100 \mu \mathrm{L}$ de sobrenadante da cultura de cada isolado e $1 \mu \mathrm{L}$ de cultura da bactéria indicadora. Como controle, o sobrenadante foi substituído por $100 \mu \mathrm{L}$ de caldo MRS. As densidades óticas (DO) foram lidas após 0, 2, 4, 6 e 8 horas de incubação a $37^{\circ} \mathrm{C}$ em espectrofotômetro, utilizando comprimento de onda de 
$540 \mathrm{~nm}$. Os testes foram realizados em triplicata.

$\mathrm{O}$ pH de cada sobrenadante foi medido após a filtragem usando fitas indicadoras de $\mathrm{pH}$ $\left(\right.$ MERCK $\left.^{\circledR}\right)$ e neutralizado $(\mathrm{pH} 7,0 \pm 0,1)$ com solução de $\mathrm{NaOH} 10 \mathrm{~N}$. O teste foi repetido, conforme descrito anteriormente, com os sobrenadantes que apresentaram atividade antimicrobiana.

Os resultados dos testes com sobrenadantes foram avaliados através do teste de Tukey $(\mathrm{P}<0,05)$ com uso do programa Statistix ${ }^{\circledR(16)}$.

\section{Resultados e Discussão}

Todos os 60 microrganismos isolados de grãos de kefir testados por meio do teste do antagonismo demonstraram capacidade de inibir pelo menos uma das bactérias indicadoras usadas. Os sobrenadantes de 25 desses isolados apresentaram atividade antimicrobiana e três mantiveram a

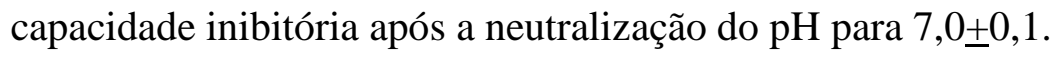

Todos os isolados foram identificados como pertencentes ao gênero Lactobacillus. A diferença nas medidas das zonas de inibição dos isolados é sugestiva de que os microrganismos pertencem a diferentes espécies ou linhagens.

O número de isolados com média de halos com $1 \mathrm{~mm}$ ou mais foi 59 para E. coli, 58 para $S$. Typhimurium e $S$. Enteritidis, 56 para $S$. aureus e 52 para L. monocytogenes. Cinquenta e um (85\%) isolados apresentaram atividade inibitória contra os cinco patógenos. Ação antagônica de Lactobacillus spp. frente a $S$. Typhimurium, $S$. Enteritidis e L. monocytogenes, utilizando o mesmo método do antagonismo, também foi verificada por Lima et. al. ${ }^{(17)}$.

Os resultados demonstram que a sensibilidade às substâncias produzidas pelas bactérias lácticas e liberadas no meio extracelular varia de acordo com o patógeno e com o isolado dos grãos de kefir. Muitas espécies de Lactocbacillus são capazes de produzir uma variedade de compostos antimicrobianos, como ácidos orgânicos, dióxido de carbono, etanol, polissacarídeos e bacteriocinas que apresentam potencial no controle de patógenos e bactérias de deterioração durante a produção e armazenamento dos alimentos $^{(18)}$. As médias dos halos de inibição observadas contra $S$. Enteritidis, S. aureus, E. coli, L. monocytogenes e $S$. Typhimurium foram, respectivamente, 5,0 mm, 4,7 mm, 4,1 $\mathrm{mm}, 3,9 \mathrm{~mm}$ e 3,6 mm. Em estudo similar, Santos et al. ${ }^{(19)}$, trabalhando com microrganismos de grãos de kefir, isolaram Lactobacillus com atividade antimicrobiana contra E. coli, L. monocytogenes, $S$. Typhimurium e $S$. Enteritidis. Diferentemente do nosso trabalho, esses autores observaram, no teste do antagonismo, maiores halos de inibição contra L. monocytogenes, o que pode se dever ao uso de espécies ou cepas de Lactobacillus distintas.

Quando adicionadas de sobrenadantes de culturas de isolados de grãos de kefir, $25(41,67 \%)$ culturas das bactérias indicadoras $S$. Typhimurium, $S$. Enteritidis, S. aureus e. coli (Tabela 1) apresentaram valores de densidades óticas estatisticamente diferentes $(\mathrm{P}<0,05)$ dos controles (culturas sem sobrenadantes), após 6 e/ou 8 horas de incubação, indicando atividade inibitória dos isolados. Não houve diferença significativa entre as DO lidas nas horas 0,2 e 4 . Ação inibitória contra $L$. monocytogenes não foi observada. Sobrenadantes de três (12\%) isolados, LIPOA 5075, LIPOA 5088 e LIPOA 5098, mantiveram a atividade antimicrobiana nos testes com sobrenadantes com $\mathrm{pH}$ neutralizado. Os valores de $\mathrm{pH}$ dos sobrenadantes, antes da neutralização, variaram entre 4,0 e 5,0 (resultados não mostrados). 
Tabela 1. Densidades ópticas $\left(\mathrm{A}_{540 \mathrm{~nm}}\right)$ das culturas das bactérias indicadoras que apresentaram valores estatisticamente diferentes $(\mathrm{P}<0,05)$ dos controles (culturas sem sobrenadantes), quando adicionadas de sobrenadantes de culturas de isolados de grãos de kefir

\begin{tabular}{|c|c|c|c|c|c|c|c|}
\hline \multirow{3}{*}{ Isolados } & \multicolumn{2}{|c|}{ E. coli } & \multirow{3}{*}{$\begin{array}{c}S . \\
\text { Enteritidis } \\
\mathbf{8 ~ h} \\
\text { Média } \\
\text { (DP) }\end{array}$} & \multicolumn{2}{|c|}{$S$. Typhimurium } & \multicolumn{2}{|c|}{ S. aureus } \\
\hline & $6 \mathrm{~h}$ & $8 \mathrm{~h}$ & & $6 \mathrm{~h}$ & $8 \mathrm{~h}$ & $6 \mathrm{~h}$ & $8 \mathrm{~h}$ \\
\hline & $\begin{array}{c}\text { Média }^{1} \\
\left(\mathrm{DP}^{2}\right)\end{array}$ & $\begin{array}{c}\text { Média } \\
\text { (DP) }\end{array}$ & & $\begin{array}{c}\text { Média } \\
\text { (DP) }\end{array}$ & $\begin{array}{c}\text { Média } \\
\text { (DP) }\end{array}$ & $\begin{array}{c}\text { Média } \\
\text { (DP) }\end{array}$ & $\begin{array}{c}\text { Média } \\
\text { (DP) }\end{array}$ \\
\hline 5021 & $\mathrm{SDE}^{3 \mathrm{a}}$ & $\mathrm{SDE}^{\mathrm{a}}$ & $\mathrm{SDE}^{\mathrm{a}}$ & $\mathrm{SDE}^{\mathrm{a}}$ & $\mathrm{SDE}^{\mathrm{a}}$ & $0,07(0,05)^{b}$ & $0,41(0,52)^{b}$ \\
\hline 5024 & $\mathrm{SDE}^{\mathrm{a}}$ & $\mathrm{SDE}^{\mathrm{a}}$ & $\mathrm{SDE}^{\mathrm{a}}$ & $0,37(0,31)^{\mathrm{b}}$ & $0,48(0,44)^{b}$ & $\mathrm{SDE}^{\mathrm{a}}$ & $\mathrm{SDE}^{\mathrm{a}}$ \\
\hline 5033 & $\mathrm{SDE}^{\mathrm{a}}$ & $\mathrm{SDE}^{\mathrm{a}}$ & $\mathrm{SDE}^{\mathrm{a}}$ & $0,34(0,19)^{\mathrm{b}}$ & $0,38(0,16)^{b}$ & $\mathrm{SDE}^{\mathrm{a}}$ & $\mathrm{SDE}^{\mathrm{a}}$ \\
\hline 5048 & $\mathrm{SDE}^{\mathrm{a}}$ & $\mathrm{SDE}^{\mathrm{a}}$ & $\mathrm{SDE}^{\mathrm{a}}$ & $\mathrm{SDE}^{\mathrm{a}}$ & $\mathrm{SDE}^{\mathrm{a}}$ & $0,42(0,36)^{b}$ & $0,45(0,36)^{b}$ \\
\hline 5058 & $\mathrm{SDE}^{\mathrm{a}}$ & $\mathrm{SDE}^{\mathrm{a}}$ & $\mathrm{SDE}^{\mathrm{a}}$ & $\mathrm{SDE}^{\mathrm{a}}$ & $\mathrm{SDE}^{\mathrm{a}}$ & $0,24(0,32)^{b}$ & $0,28(0,30)^{b}$ \\
\hline 5065 & $\mathrm{SDE}^{\mathrm{a}}$ & $\mathrm{SDE}^{\mathrm{a}}$ & $\mathrm{SDE}^{\mathrm{a}}$ & $0,38(0,19)^{\mathrm{b}}$ & $0,45(0,18)^{b}$ & $\mathrm{SDE}^{\mathrm{a}}$ & $\mathrm{SDE}^{\mathrm{a}}$ \\
\hline 5075 & $0,17(0,17)^{b}$ & $0,07(0,12)^{b}$ & $0,08(0,11)^{b}$ & $0,15(0,13)^{\mathrm{b}}$ & $0,16(0,15)^{b}$ & $0,13(0,12)^{b}$ & $0,12(0,13)^{b}$ \\
\hline 5077 & $0,24(0,28)^{b}$ & $0,07(0,11)^{b}$ & $0,05(0,06)^{b}$ & $0,11(0,14)^{b}$ & $0,09(0,15)^{b}$ & $0,09(0,09)^{b}$ & $0,08(0,12)^{b}$ \\
\hline 5078 & $\mathrm{SDE}^{\mathrm{a}}$ & $0,07(0,12)^{b}$ & $0,05(0,09)^{b}$ & $0,15(0,19)^{\mathrm{b}}$ & $0,15(0,22)^{b}$ & $0,12(0,08)^{b}$ & $0,12(0,10)^{b}$ \\
\hline 5079 & $\mathrm{SDE}^{\mathrm{a}}$ & $0,06(0,11)^{b}$ & $0,05(0,07)^{b}$ & $0,08(0,09)^{\mathrm{b}}$ & $0,08(0,14)^{b}$ & $0,12(0,12)^{b}$ & $0,13(0,14)^{b}$ \\
\hline 5080 & $0,27(0,33)^{b}$ & $0,06(0,10)^{b}$ & $0,04(0,07)^{b}$ & $0,10(0,12)^{b}$ & $0,08(0,15)^{b}$ & $0,11(0,10)^{b}$ & $0,11(0,11)^{b}$ \\
\hline 5081 & $0,22(0,28)^{b}$ & $0,05(0,09)^{\mathrm{b}}$ & $0,04(0,06)^{b}$ & $0,10(0,11)^{\mathrm{b}}$ & $0,10(0,14)^{b}$ & $0,10(0,09)^{b}$ & $0,10(0,08)^{b}$ \\
\hline 5082 & $\mathrm{SDE}^{\mathrm{a}}$ & $0,07(0,12)^{b}$ & $0,03(0,05)^{b}$ & $0,12(0,14)^{b}$ & $0,11(0,16)^{b}$ & $0,10(0,11)^{b}$ & $0,09(0,12)^{b}$ \\
\hline 5084 & $\mathrm{SDE}^{\mathrm{a}}$ & $\mathrm{SDE}^{\mathrm{a}}$ & $\mathrm{SDE}^{\mathrm{a}}$ & $0,39(0,26)^{b}$ & $0,47(0,26)^{b}$ & $0,32(0,36)^{b}$ & $0,42(0,46)^{b}$ \\
\hline 5087 & $\mathrm{SDE}^{\mathrm{a}}$ & $0,11(0,10)^{b}$ & $0,05(0,09)^{\mathrm{b}}$ & $0,10(0,12)^{\mathrm{b}}$ & $0,10(0,14)^{b}$ & $0,11(0,06)^{b}$ & $0,10(0,08)^{b}$ \\
\hline 5088 & $0,26(0,32)^{b}$ & $0,09(0,09)^{\mathrm{b}}$ & $0,04(0,07)^{b}$ & $0,10(0,14)^{\mathrm{b}}$ & $0,09(0,16)^{b}$ & $0,14(0,14)^{b}$ & $0,11(0,16)^{b}$ \\
\hline 5091 & $0,13(0,15)^{b}$ & $0,04(0,07)^{b}$ & $0,03(0,05)^{b}$ & $0,06(0,10)^{b}$ & $0,07(0,12)^{b}$ & $0,11(0,10)^{b}$ & $0,09(0,13)^{b}$ \\
\hline 5092 & $\mathrm{SDE}^{\mathrm{a}}$ & $0,14(0,14)^{b}$ & $0,04(0,06)^{b}$ & $0,07(0,11)^{\mathrm{b}}$ & $0,07(0,12)^{b}$ & $0,11(0,11)^{b}$ & $0,09(0,11)^{b}$ \\
\hline 5093 & $\mathrm{SDE}^{\mathrm{a}}$ & $\mathrm{SDE}^{\mathrm{a}}$ & $\mathrm{SDE}^{\mathrm{a}}$ & $0,36(0,21)^{\mathrm{b}}$ & $0,78(0,38)^{b}$ & $\mathrm{SDE}^{\mathrm{a}}$ & $\mathrm{SDE}^{\mathrm{a}}$ \\
\hline 5094 & $\mathrm{SDE}^{\mathrm{a}}$ & $\mathrm{SDE}^{\mathrm{a}}$ & $\mathrm{SDE}^{\mathrm{a}}$ & $0,33(0,19)^{\mathrm{b}}$ & $0,54(0,03)^{b}$ & $\mathrm{SDE}^{\mathrm{a}}$ & $\mathrm{SDE}^{\mathrm{a}}$ \\
\hline 5095 & $\mathrm{SDE}^{\mathrm{a}}$ & $0,14(0,12)^{b}$ & $0,04(0,06)^{b}$ & $0,07(0,10)^{\mathrm{b}}$ & $0,07(0,13)^{b}$ & $0,14(0,12)^{b}$ & $0,14(0,12)^{b}$ \\
\hline 5097 & $\mathrm{SDE}^{\mathrm{a}}$ & $0,08(0,14)^{b}$ & SDE & $0,07(0,10)^{\mathrm{b}}$ & $0,07(0,13)^{b}$ & $0,10(0,10)^{b}$ & $0,09(0,12)^{b}$ \\
\hline 5098 & $0,22(0,27)^{b}$ & $0,05(0,09)^{\mathrm{b}}$ & $0,04(0,05)^{b}$ & $0,07(0,11)^{\mathrm{b}}$ & $0,07(0,13)^{b}$ & $0,09(0,09)^{b}$ & $0,08(0,10)^{b}$ \\
\hline 5104 & $\mathrm{SDE}^{\mathrm{a}}$ & $0,07(0,09)^{\mathrm{b}}$ & $0,03(0,05)^{b}$ & $0,06(0,10)^{\mathrm{b}}$ & $0,07(0,13)^{b}$ & $0,10(0,09)^{b}$ & $0,08(0,09)^{b}$ \\
\hline 5107 & $\mathrm{SDE}^{\mathrm{a}}$ & $\mathrm{SDE}^{\mathrm{a}}$ & $0,04(0,07)^{b}$ & $0,14(0,11)^{\mathrm{b}}$ & $0,19(0,18)^{b}$ & $0,06(0,06)^{b}$ & $0,08(0,06)^{b}$ \\
\hline
\end{tabular}

Metabólitos formados durante a fermentação ou produtos de degradação podem ser responsáveis pelos efeitos inibitórios ${ }^{(20)}$. Essas substâncias podem exercer atividade antimicrobiana por diferentes mecanismos, desestabilização da membrana, lise celular, degradação de ácidos nucleicos e inibição da síntese de proteínas. Os ácidos orgânicos, lático e acético, resultantes do catabolismo dos carboidratos contribuem para o decréscimo do $\mathrm{pH}$, tornando o ambiente hostil para a maioria dos microrganismos ${ }^{(21)}$. Segundo Eklund ${ }^{(22)}$, a ação inibidora dos ácidos orgânicos é atribuída à redução direta do pH intracelular por ionização dos ácidos não dissociados que podem permear a membrana celular por difusão e liberar prótons no citoplasma celular. O influxo de prótons induz a acidificação do citoplasma e dissipa o potencial de prótons da membrana inibindo a geração 
de energia ${ }^{(23)}$. Garrote et al. ${ }^{(6)}$ demonstraram que o efeito bacteriostático do kefir sobre $E$. coli devese principalmente aos ácidos orgânicos produzidos durante o processo de fermentação. Estes autores não observaram efeito inibitório quando utilizaram sobrenadantes com $\mathrm{pH}$ neutralizado. Rivas e Rivero $^{(24)}$, estudando a atividade inibitória de Lactobacillus, observaram que o ácido láctico é o principal responsável por esse efeito. Entretanto, Garrote et al. ${ }^{(6)}$, em estudo da ação de ácidos orgânicos, concluíram que, apesar de o ácido láctico ser importante para a inibição, a presença de ácido acético potencializa o efeito inibitório. A ausência de efeito inibitório dos sobrenadantes sobre L. monocytogenes pode se dever à insuficiente diminuição do $\mathrm{pH}$, uma vez que essas bactérias são capazes de adaptação, tornando-se mais resistentes a ambientes ácidos ${ }^{(25)}$, ou à pouca quantidade de substância bioativa. O mecanismo de ação dos Lactobacillus sobre o crescimento de microrganismos patogênicos parece se dever principalmente à liberação de produtos que acidificam o meio, como ácidos orgânicos, uma vez que, dos 25 isolados cujos sobrenadantes apresentaram atividade inibitória frente aos microrganismos testados, apenas três mantiveram essa ação após a neutralização dos sobrenadantes. Entretanto, pelo menos nesses três isolados, a produção de outras substâncias bioativas com ação antimicrobiana parece ter ocorrido. A produção por alguns Lactobacillus isolados de grãos de kefir de um exopolissacarídio denominado kefiran tem sido responsabilizada por suas propriedades antimicrobianas ${ }^{(10)}$.

O conhecimento a respeito de bactérias que apresentem ação inibitória sobre microrganismos indesejáveis abre a perspectiva da sua utilização na indústria de alimentos. Além de conferir aroma, sabor e textura aos derivados lácteos, as bactérias ácido-lácticas podem ser empregadas como bioconservantes, em substituição aos conservantes tradicionais.

\section{Conclusão}

Lactobacillus isolados de grãos de kefir apresentam atividade antimicrobiana contra cepas de Samonella sorotipos Enteritidis e Typhimurium, E. coli O157:H7, S. aureus e L. monocytogenes, o que decorre provavelmente da produção de ácidos orgânicos que levam à diminuição do $\mathrm{pH}$ do meio. Entretanto, em alguns casos, esse não é o único fator responsável pelo efeito antimicrobiano, o que remete à potencial utilização dessas bactérias ácido-lácticas ou seus metabólitos em alimentos como forma alternativa de controle de microrganismos indesejáveis.

\section{Agradecimentos}

Os autores agradecem ao Conselho Nacional de Desenvolvimento Científico e Tecnológico (CNPq) pelo aporte financeiro ao estudo.

\section{Referências}

1 Martinez JL, Baquero F. Interactions among strategies associated with bacterial infection: pathogenicity, epidemicity, and antibiotic resisteance. Clinic Microbiology Reviews. 2002;15(4):647-679. Disponível em: http://cmr.asm.org/content/15/4/647.

2 Irigoyen A, Arana I, Castiella M, Torre P, Ibáñez FC. Microbiological, physicochemical and sensory characteristics of kefir during storage. Food Chemistry. 2005;90(4):613-620. Disponível em: 
doi:10.1016/j.foodchem.2004.04.021.

3 Garrote GL, Abraham AG, De Antoni GL. Chemical and microbiological characterisation of kefir grains. Journal of Dairy Research. 2001;68(4):639-652. Disponível em: http://dx.doi.org/10.1017/S0022029901005210.

4 Witthuhn RC, Schoeman T, Britz TJ. Isolation and characterization of the microbial population of different South African kefir grains. International Journal of Dairy Technology. 2004;57(1):33-37. Disponível em: http://onlinelibrary.wiley.com/doi/10.1111/j.1471-0307.2004.00126.x/full.

5 Chifiriuc MC, Cioaca AC, Lazar V. In vitro assay of the antimicrobial activity of kephir against bacterial and fungal strains. Anaerobe. 2011;17(6):433-435. Disponível em: http://www.sciencedirect.com/science/article/pii/S1075996411000886.

6 Garrote GL, Abraham AG, De Antoni GL. Inhibitory power of kefir: the role of organic acids. Journal of Food Protection. 2000;63(3):364-369. Disponível em: http://www.ingentaconnect.com/content/iafp/jfp/2000/00000063/00000003/art00012.

7 Rahimzadeh G, Fazeli MR, Mozafari A, Mesbahi M. Evaluation of anti-microbial activity and wound healing of kefir. International Journal of Pharmaceutical Sciences and Research. 2015;6(1)286-293. Disponível em: http://search.proquest.com/openview/9ba6f3b6fbafea9ecdf8b3f6d5e92c9a/1?pq-origsite=gscholar.

8 Leite AMO, Miguel MAL, Peixoto RS, Ruas-Madiedo P, Paschoalin VMF, Mayo B, Delgado S. Probiotic potential of selected lactic acid bacteria strains isolated from Brazilian kefir grains. Journal of Dairy Science. 2015;98(6):3622-3632. Disponível

http://www.sciencedirect.com/science/article/pii/S0022030215002295.

9 Shokryazdan P, Sieo CC, Kalavathy R, Liang JB, Alitheen NB, Jahromi MF, Ho YW. Probiotic potential of Lactobacillus strains with antimicrobial activity against some human pathogenic strains. BioMed Research International. 2014;2014:Article ID 927268. Disponível em: http://dx.doi.org/10.1155/2014/927268.

10 Rodrigues KL, Caputo LRG, Carvalho JCT, Evangelista J, Schneedorf JM. Antimicrobial and healing activity of kefir and kefiran extract. International Journal of Antimicrobiol Agents. 2005;25(5):404-408. Disponível em: http://dx.doi.org/10.1016/j.ijantimicag.2004.09.020.

11 Powell JE, Witthuhn RC, Todorov, SD, Dicks LMT. Characterization of bacteriocin ST8KF produced by a kefir isolate Lactobacillus plantarum ST8KF. International Dairy Journal. 2007;17(3):190-198. Disponível em: http://dx.doi.org/10.1016/j.idairyj.2006.02.012.

12 Barrow GI, Feltham RKA. Manual for identification of medical bacterial. $3^{\text {rd }}$ ed. Cambridge: Cambridge University, 1993.

13 Jacobsen CN, Rosenfeldt Nielsen V, Hayford AE, Møller PL, Michaelsen KF, Paerregaard A, Sandström B, Tverde M, Jakobsen M. Screening of Probiotic Activities of forty-seven strains of Lactobacillus spp. by in vitro techniques and evaluation of the colonization ability of five selected strains in humans. Applied and Environmental Microbiology. 1999;65(11):4949-4956. Disponível em: http://aem.asm.org/content/65/11/4949.short.

14 Lin WH, Hwang CF, Chen LW, Tsen HY. Viable counts, characteristic evaluation for commercial lactic acidic bacteria products. Food Microbiology. 2006;23(1):74-81. Disponível em: http://dx.doi.org/10.1016/j.fm.2005.01.013.

15 Krüger CCH, Silva CA, Vedana MIS, Tiene C, Cândido LMB. Atividade antimicrobiana de peptídeos obtidos de caseína bovina. Alimentos e Nutrição. 2006;17(1):7-12. Disponível em: http://servbib.fcfar.unesp.br/seer/index.php/alimentos/article/viewFile/110/123.

16 Statistix $^{\circledR}$. Statistix 8 Analytical Software. User’s manual. Tallahassee. FL. 2003. 396p.

17 Lima ET, Andreatti Filho RL, Okamoto AS, Noujaim JC, Barros MR, Crocci AJ. Evaluation in vitro of the antagonistic substances produced by Lactobacillus spp. isolated from chickens. Canadian Journal of Veterinary Research. 2007;71(2):103-107. Disponível em: http://www.ncbi.nlm.nih.gov/pmc/articles/PMC1829185/.

18 Messes W, De Vuyst L. Inhibitory substances produced by lactobacilli isolated from sourdoughs - a review. International Journal of Food Microbiology. 2002;72(1-2):31-43. Disponível em: 


\section{http://dx.doi.org/10.1016/S0168-1605(01)00611-0.}

19 Santos A, San Mauro M, Sanchez A, Torres JM, Marquina D. The antimicrobial properties of different strains of Lactobacillus spp. isolated from kefir. Systematic and Applied Microbiology. 2003;26(3):434-443. Disponível em: http://dx.doi.org/10.1078/072320203322497464.

20 Farnworth ER. Kefir - a complex probiotic. In: Gibson GR. Food Science \& Technology Bulletin: Functional Foods. Readin: IFIS; 2005. p.1-17.

21 Bosch A, Golowczyc MA, Abraham AG, GARROTE GL, De Antoni GL, Yantorno O. Rapid discrimination of lactobacilli isolated from kefir grains by FT-IR espectroscopy. International Journal of Food Microbiology. 2006;111(3)280-287. Disponível em: http://www.sciencedirect.com/science/article/pii/S0168160506003114.

22 Eklund T. The antimicrobial effect of dissociated and undissociated sorbic acid at different pH levels. Journal of Applied Bacteriology. 1983;54:383-389. Disponível em: http://onlinelibrary.wiley.com/doi/10.1111/j.1365-2672.1983.tb02632.x/full.

23 Diez-Gonzales F, Russel JB. The ability of Escherichia coli $\mathrm{O} 157: \mathrm{H} 7$ to decrease its intracellular pH and resist the toxicity of acetic acid. Microbiology. 1997;143(4):1175-1180. Disponível em: http://dx.doi.org/10.1099/00221287-143-4-1175.

24 Rivas CCA, Rivero CGD. Efecto antagónico de Lactobacillus plantarum aislado de pastizal de finca lechera. Respyn Revista de Salud Pública y Nutrición. 2009;10(1). Disponível em: http://www.medigraphic.com/pdfs/revsalpubnut/spn-2009/spn091d.pdf.

25 Van Schaik W, Gahan CGM, Hill C. Acid-adapted Listeria monocytogenes displays enhanced tolerance against the lantibiotics nisin and lacticin 3147. Journal of Food Protection. 1999;62(5):536-539. Disponível em: http://www.ingentaconnect.com/content/iafp/jfp/1999/00000062/00000005/art00018. 